\title{
Bayesian and maximum likelihood estimation of entropy of the inverse Weibull distribution under generalized type I progressive hybrid censoring
}

\author{
Kyeongjun Lee ${ }^{1, a}$ \\ ${ }^{a}$ Division of Mathematics and Big Data Science, Daegu University, Korea
}

\begin{abstract}
Entropy is an important term in statistical mechanics that was originally defined in the second law of thermodynamics. In this paper, we consider the maximum likelihood estimation (MLE), maximum product spacings estimation (MPSE) and Bayesian estimation of the entropy of an inverse Weibull distribution (InW) under a generalized type I progressive hybrid censoring scheme (GePH). The MLE and MPSE of the entropy cannot be obtained in closed form; therefore, we propose using the Newton-Raphson algorithm to solve it. Further, the Bayesian estimators for the entropy of InW based on squared error loss function (SqL), precautionary loss function $(\mathrm{PrL})$, general entropy loss function $(\mathrm{GeL})$ and linex loss function $(\mathrm{LiL})$ are derived. In addition, we derive the Lindley's approximate method (LiA) of the Bayesian estimates. Monte Carlo simulations are conducted to compare the results among MLE, MPSE, and Bayesian estimators. A real data set based on the GePH is also analyzed for illustrative purposes.
\end{abstract}

Keywords: generalized type I progressive hybrid censoring, inverse Weibull distribution, Lindley approximation, maximum likelihood estimation, maximum product spacings estimation

\section{Introduction}

The inverse Weibull distribution (InW) can be readily applied to a wide range of situations including applications in reliability and ecology. The cumulative distribution function (cdf) and probability density function (pdf) of InW are given by

$$
F(x ; \alpha, \beta)=\exp \left(-\beta x^{-\alpha}\right), \quad x>0, \alpha>0, \beta>0,
$$

and

$$
f(x ; \alpha, \beta)=\alpha \beta x^{-(\alpha+1)} \exp \left(-\beta x^{-\alpha}\right), \quad x>0, \alpha>0, \beta>0,
$$

where $\alpha$ and $\beta$ are the scale and shape parameters, respectively. Note that when $\beta=1$, the $\operatorname{InW}$ is referred to as the inverse exponential. Keller et al. (1985) derived the InW by investigating failures of mechanical components subject to degradation. Calabria and Pulcini (1990) derived the least squared estimates (LSE) and maximum likelihood estimation (MLE) of the parameters of the InW. They also derived the Bayesian estimator of the parameters as well as confidence intervals for tolerance limits and reliability.

\footnotetext{
${ }^{1}$ Division of Mathematics and Big Data Science, Daegu University, Gyeongsan-si 38453, Korea.

E-mail: indra_74@naver.com
}

Published 31 July 2020 / journal homepage: http://csam.or.kr

(C) 2020 The Korean Statistical Society, and Korean International Statistical Society. All rights reserved. 
Entropy is an important term in statistical mechanics that was originally defined in the second law of thermodynamics. Shannon (1948) re-defined entropy in an information theorem using the concepts of probability and statistics. The differential entropy $H(f)$ of the random variable $X$ is defined to be

$$
H(f)=-\int_{-\infty}^{\infty} f(x) \log f(x) d x
$$

where $f(x)$ denote a probability density function (Cover and Thomas, 2005). Baratpour et al. (2007) considered lower/upper bounds for entropy of upper record values. They provided the entropy of upper record values. Kang et al. (2012) considered the estimators of entropy of a double exponential distribution based on multiply type II censoring. Cho et al. (2014) provided the estimators of entropy of a Rayleigh distribution based on the doubly-generalized type II hybrid censoring scheme. Cho et al. (2015a) provided the estimators of entropy of a Weibull distribution based on a generalized progressive hybrid censoring scheme. Lee (2017) considered the Bayesian estimators of entropy of the InW under generalized progressive hybrid censoring scheme.

This paper compares the MLE and maximum product spacings estimation (MPSE) of entropy of the InW under generalized type I progressive hybrid censoring scheme $(\mathrm{GePH})$ in a classical method. In addition, we derived the Bayesian estimation of entropy based on variety of loss functions (the squared error loss function (SqL), precautionary loss function (PrL), general entropy loss function $(\mathrm{GeL})$ and linex loss function (LiL)) to compare the research of Lee (2017). We derive Bayesian estimators using the Lindley's approximate method (LiA) as well as compare Bayesian estimators using the Tierney and Kadane approximation method (TiK). In Section 2, we derive the MLE, MPSE, and Bayesian estimators of the entropy of the InW based on the GePH. In Section 3, Monte Carlo simulations are conducted to compare the results of MLE, MPSE and Bayesian estimators, and a real data set based on the GePH are analyzed for illustrative purposes.

\section{Entropy estimation}

Let $X$ be a random variable with the cdf, $F(x)$, and the pdf, $f(x)$. Then, the differential entropy of the $\mathrm{InW}$ is given by (Lee, 2017)

$$
H(f)=E[-\log f(x)]=-\alpha \beta[A+B+C],
$$

where

$$
A=\frac{\log (\alpha \beta)}{\alpha \beta}, \quad B=-\frac{\alpha+1}{\alpha^{2} \beta}(\gamma+\log \beta), \quad C=-\frac{1}{\alpha \beta}
$$

and $\gamma$ is the Euler-Mascheroni constant.

\subsection{Maximum likelihood estimation}

In this subsection, we derive the MLEs of the unknown parameters of the InW as well as obtain the MLE of entropy. Suppose that $X_{1}, X_{2}, \ldots, X_{n}$ denote the corresponding life-times from a probability distribution with pdf $f(x)$ and cdf $F(x)$. The integer $m, k \in\{1,2, \ldots, n\}$ are pre-fixed such that $k<m$. The progressive censoring scheme $\left(R_{1}, R_{2}, \ldots, R_{m}\right)$ are pre-fixed integers satisfying $\sum_{i=1}^{m} R_{i}+m=n$. And $T \in(0, \infty)$ is a pre-fixed time point. The likelihood functions of the GePH (Cho et al., 2015b) 
are then given by

$$
\begin{aligned}
& \text { Case I: } L_{1}(\alpha, \beta)=C_{1} \prod_{i=1}^{k-1} f\left(x_{i: m: n}\right)\left[1-F\left(x_{i: m: n}\right)\right]^{R_{i}} f\left(x_{k: m: n}\right)\left[1-F\left(x_{k: m: n}\right)\right]^{R_{k}^{k}}, \\
& \text { Case II: } L_{2}(\alpha, \beta)=C_{2} \prod_{i=1}^{D} f\left(x_{i: m: n}\right)\left[1-F\left(x_{i: m: n}\right)\right]^{R_{i}}[1-F(T)]^{R_{D+1}^{*}}, \\
& \text { Case III: } L_{3}(\alpha, \beta)=C_{3} \prod_{i=1}^{m} f\left(x_{i: m: n}\right)\left[1-F\left(x_{i: m: n}\right)\right]^{R_{i}}
\end{aligned}
$$

where $D$ denote the number of observed failures up to time $T, C_{1}=\left[\prod_{i=1}^{k} \sum_{k=i}^{m}\left(R_{k}+1\right)\right], C_{2}=$ [ $\left.\prod_{i=1}^{D} \sum_{k=i}^{m}\left(R_{k}+1\right)\right], C_{3}=\left[\prod_{i=1}^{m} \sum_{k=i}^{m}\left(R_{k}+1\right)\right], R_{k}^{*}=n-k-\sum_{i=1}^{k-1} R_{i}$, and $R_{D+1}^{*}=n-D-\sum_{i=1}^{D} R_{i}$. Utilizing Equations (1.1) and (1.2), the likelihood functions of $\alpha$ and $\beta$ are given by

$$
\begin{aligned}
& \text { Case I: } L_{1}(\alpha, \beta)=C_{1}(\alpha \beta)^{k} x_{k: m: n}^{-(\alpha+1)} e^{-\beta x_{k: m: n}^{-\alpha}}\left[1-e^{\left.-\beta x_{k: m}^{-\alpha}\right]^{1}}\right]^{R_{k}^{k}} \prod_{i=1}^{k-1} x_{i: m: n}^{-(\alpha+1)} e^{-\beta x_{i: m: n}^{-\alpha}}\left[1-e^{-\beta x_{i: m: n}^{-\alpha}}\right]^{R_{i}}, \\
& \text { Case II: } L_{2}(\alpha, \beta)=C_{2}(\alpha \beta)^{D} \prod_{i=1}^{D} x_{i: m: n}^{-(\alpha+1)} e^{-\beta x_{i: m: n}^{-\alpha}}\left[1-e^{-\beta x_{i: m: n}^{-\alpha}}\right]^{R_{i}}\left[1-e^{-\beta T^{-\alpha}}\right]^{R_{D+1}^{*}}, \\
& \text { Case III: } L_{3}(\alpha, \beta)=C_{3}(\alpha \beta)^{m} \prod_{i=1}^{m} x_{i: m: n}^{-(\alpha+1)} e^{-\beta x_{i: m n n}^{-\alpha}}\left[1-e^{\left.-\beta x_{i: m: n}^{-\alpha}\right]^{R_{i}} .}\right.
\end{aligned}
$$

Therefore, the likelihood function of the GePH based on the InW is given by

$$
L(\alpha, \beta) \propto(\alpha \beta)^{s} \prod_{i=1}^{s} x_{i: m: n}^{-(\alpha+1)} e^{-\beta x_{i m n n}^{-\alpha}}\left[1-e^{-\beta x_{i m n n}^{-\alpha}}\right]^{R_{i}} \eta(\alpha, \beta),
$$

where $s=k, \eta(\alpha, \beta)=1$ and $R_{k}=n-k-\sum_{i=1}^{k-1} R_{i}$ for Case I, $s=D$ and $\eta(\alpha, \beta)=\left[1-e^{-\beta T^{-\alpha}}\right]^{R_{D+1}^{*}}$ for Case II, and $s=m$ and $\eta(\alpha, \beta)=1$ for Case III. Hence, the log-likelihood function becomes

$$
l(\alpha, \beta) \propto s \log (\alpha \beta)-(\alpha+1) \sum_{i=1}^{s} \log x_{i: m: n}-\beta \sum_{i=1}^{s} x_{i: m: n}^{-\alpha}+\sum_{i=1}^{s} R_{i} \log \left[1-e^{-\beta x_{i: m: n}^{-\alpha}}\right]+\log [\eta(\alpha, \beta)] .
$$

Differentiating the $\log$-likelihood function partially with respect to $\alpha$ and $\beta$ and then equating to zero, we have

$$
\frac{\partial l(\alpha, \beta)}{\partial \alpha}=\frac{s}{\alpha}+\sum_{i=1}^{s}\left[\beta x_{i: m: n}^{-\alpha} \log x_{i: m: n}-\log x_{i: m: n}-R_{i} \frac{x_{i: m: n}^{-\alpha} \log x_{i: m: n} e^{-\beta x_{i: m: n}^{-\alpha}}}{1-e^{-\beta x_{i: n: n}^{-\alpha}}}\right]+\eta_{\alpha}^{1}(\alpha, \beta)=0,
$$

and

$$
\frac{\partial l(\alpha, \beta)}{\partial \beta}=\frac{s}{\beta}-\sum_{i=1}^{s} x_{i: m: n}^{-\alpha}+\sum_{i=1}^{s} R_{i} \frac{x_{i: m: n} e^{-\beta x_{i: m: n}^{-\alpha}}}{1-e^{-\beta x_{i: m: n}}}+\eta_{\beta}^{1}(\alpha, \beta)=0,
$$


where, for Case II,

$$
\eta_{\alpha}^{1}(\alpha, \beta)=-R_{D+1}^{*} \frac{\beta T^{-\alpha} \log T e^{-\beta T^{-\alpha}}}{1-e^{-\beta T^{-\alpha}}} \quad \text { and } \quad \eta_{\beta}^{1}(\alpha, \beta)=R_{D+1}^{*} \frac{T^{-\alpha} e^{-\beta T^{-\alpha}}}{1-e^{-\beta T^{-\alpha}}},
$$

for Case I and Case III,

$$
\eta_{\alpha}^{1}(\alpha, \beta)=\eta_{\beta}^{1}(\alpha, \beta)=0
$$

The MLEs of $\alpha$ and $\beta$ are the solution of Equations (2.4) and (2.5). However, solutions for $\alpha$ and $\beta$ cannot be obtained explicitly. Therefore, we propose to use the Newton-Raphson algorithm to solve the number. Using the MLEs of $\alpha$ and $\beta$, say $\hat{\alpha}$ and $\hat{\beta}$, the MLE of the entropy function (2.1) is obtained as

$$
\hat{H}=1+\left(1+\frac{1}{\hat{\alpha}}\right)(\gamma+\log \hat{\beta})-\log (\hat{\alpha} \hat{\beta})
$$

\subsection{Maximum product spacings estimation}

The method of MLE works well if each of the contributions to the likelihood function is bounded above. Various authors have noted the limitations of MLE in different contexts. Its greatest limitation is that it cannot work for a heavy tailed continuous distribution with unknown location and scale parameters. It also creates problems in mixture of continuous distributions and in such cases where MLE method can break down. Further they reiterated the view of Huzurbazar (1948) that no stationary point (and hence no local maximum) can provide a consistent estimator, when the concerned distribution is a J-shape, as in the case of the Weibull and gamma distributions when the shape parameter is less than unity.

Cheng and Amin (1983) introduced the MPSE as an alternative to MLE of parameters in order to overcome the shortcomings and have better applicability in situations that possesses properties similar to MLE. They proposed to replace the likelihood function by product of spacings (PSs) and claimed that it retains most of the properties of the MLE.

For the GePH, the PS to be maximized is

$$
S(\alpha, \beta) \propto \prod_{i=1}^{s+1}\left[F\left(x_{i: m: n}\right)-F\left(x_{i-1: m: n}\right)\right] \prod_{i=1}^{s}\left[1-F\left(x_{i: m: n}\right)\right]^{R_{i}} \eta(\alpha, \beta),
$$

where $F\left(x_{0: m: n}\right) \equiv 0, F\left(x_{s+1: m: n}\right) \equiv F(T) \equiv \xi$ for Cases I and II, and $F\left(x_{s+1: m: n}\right) \equiv \xi \equiv 1$ for Case III. Then, taking the logarithm of $S(\alpha, \beta)$, we get

$$
\begin{aligned}
\log S(\alpha, \beta) \propto & -\beta x_{1: m: n}^{-\alpha}+\sum_{i=2}^{s} \log \left[e^{-\beta x_{i: m: n}^{-\alpha}}-e^{-\beta x_{i-1: m: n}^{-\alpha}}\right]+\log \left[\xi-e^{-\beta x_{s: m: n}^{-\alpha}}\right] \\
& +\sum_{i=1}^{s} R_{i} \log \left[1-e^{-\beta x_{i: m: n}^{-\alpha}}\right]+\log [\eta(\alpha, \beta)] .
\end{aligned}
$$

After differentiating the above equation with respect to parameters and then equating it to zero, we 
have

$$
\begin{aligned}
& \frac{\partial \log S(\alpha, \beta)}{\partial \alpha}=-\beta x_{1: m: n}^{-\alpha} \log x_{1: m: n}+\beta \sum_{i=2}^{s} \frac{x_{i: m: n}^{-\alpha} \log x_{i: m: n} e^{-\beta x_{i: m: n}^{-\alpha}-x_{i-1: m: n}^{-\alpha} \log x_{i-1: m: n} e^{-\beta x_{i-1: m: n}^{-\alpha}}}}{e^{-\beta x_{i: m: n}^{-\alpha}}-e^{-\beta x_{i-1: m: n}^{-\alpha}}} \\
& -\frac{\beta x_{s: m: n}^{-\alpha} \log x_{s: m: n} e^{-\beta x_{s: m: n}^{-\alpha}}}{\xi-e^{-\beta x_{s: m: n}^{-\alpha}}}-\sum_{i=1}^{s} R_{i} \frac{\beta x_{i: m: n}^{-\alpha} \log x_{i: m: n} e^{-\beta x_{i: m: n}^{-\alpha}}}{1-e^{-\beta x_{i: m: n}^{-\alpha}}}+\eta_{\alpha}^{1}(\alpha, \beta)=0
\end{aligned}
$$

and

$$
\begin{aligned}
\frac{\partial \log S(\alpha, \beta)}{\partial \beta}= & -x_{1: m: n}^{-\alpha}-\sum_{i=2}^{s} \frac{x_{i: m: n}^{-\alpha} e^{-\beta x_{i: m: n}^{-\alpha}}-x_{i-1: m: n}^{-\alpha} e^{-\beta x_{i-1: m: n}^{-\alpha}}}{e^{-\beta x_{i: m: n}^{-\alpha}}-e^{-\beta x_{i-1: m: n}^{-\alpha}}}+\frac{x_{s: m: n}^{-\alpha} e^{-\beta x_{s: m: n}^{-\alpha}}}{\xi-e^{-\beta x_{s: m: n}^{-\alpha}}} \\
& +\sum_{i=1}^{s} R_{i} \frac{x_{i: m: n}^{-\alpha} e^{-\beta x_{i: m: n}^{-\alpha}}}{1-e^{-\beta x_{i: m: n}^{-\alpha}}}+\eta_{\beta}^{1}(\alpha, \beta)=0 .
\end{aligned}
$$

The MPSEs of $\alpha$ and $\beta$ are the solutions of Equations (2.7) and (2.8), respectively. However, solutions for $\alpha$ and $\beta$ are not available. Therefore, we propose to use the Newton-Raphson algorithm to solve it. Using the MPSEs of $\alpha$ and $\beta$, say $\tilde{\alpha}$ and $\tilde{\beta}$, the MPSE of entropy function is obtained as

$$
\tilde{H}=1+\left(1+\frac{1}{\tilde{\alpha}}\right)(\gamma+\log \tilde{\beta})-\log (\tilde{\alpha} \tilde{\beta})
$$

\subsection{Bayesian estimation}

\subsubsection{Loss function}

In this subsection, we derive the Bayesian estimators of the entropy of the InW with the GePH under symmetric, as well asymmetric loss functions. A popular symmetric loss function is the SqL, the Bayesian estimates under $\mathrm{SqL}$ is the posterior mean. The $\mathrm{SqL}$ is widely employed in the Bayesian inference due to its computational simplicity. It is a symmetric loss function that gives equal weight to overestimation as well as underestimation. However, this is not a practical criterion. In estimating reliability and failure rate functions, an overestimation causes more damage than underestimation. To resolve such a situation, asymmetrical loss functions are more appropriate.

Varian (1975) introduces the LiL in response to the criticism of the SqL. The LiL is defined as $L_{2}(\hat{\theta}, \theta)=\exp [\lambda(\hat{\theta}-\theta)]-\lambda(\hat{\theta}-\theta)-1$, where $\hat{\theta}$ denote the estimator of $\theta$. The magnitude of $\lambda$ reflects the degree of symmetry while the sign of $\lambda$ reflects the direction of symmetry. The Bayesian estimator under the LiL is given by $\hat{\theta}_{L}=-1 / \lambda \log E[\exp (-\lambda \theta)]$, provided that $E[\exp (-\lambda \theta)]$ exists.

Basu and Ebrahimi (1991) find that the LiL is unsuitable to estimate the scale parameter and other quantities. Therefore, Basu and Ebrahimi (1991) define a modified LiL instead. Subsequently, Calabria and Pulcini (1994) proposed a suitable alternative to the modified LiL that is the GeL. The GeL is defined as $L_{3}(\hat{\theta}, \theta)=(\hat{\theta} / \theta)^{\lambda}-\lambda \log (\hat{\theta} / \theta)-1$. The magnitude of $\lambda$ reflects the degree of symmetry while the sign of $\lambda$ reflects the direction of symmetry. The Bayesian estimator under the GeL is given by $\hat{\theta}_{E}=E\left[\theta^{-\lambda}\right]^{-1 / \lambda}$, provided that $E\left[\theta^{-\lambda}\right]$ exists. Here, $\hat{\theta}_{E}=\hat{\theta}_{S}$ when $\lambda=-1$.

Norstrom (1996) introduces the PrL, which is defined as $L_{4}(\hat{\theta}, \theta)=(\theta-\hat{\theta})^{2} / \hat{\theta}$, and claim that it is useful to derive conservative estimators since it approaches infinity near the origin and prevents underestimation. This loss function is suitable for situations where underestimation may lead to serious consequences. The Bayesian estimator under the PrL is given by $\hat{\theta}_{P}=\sqrt{E\left(\theta^{2}\right)}$, provided that $E\left(\theta^{2}\right)$ exists. Here, $\hat{\theta}_{P}=\hat{\theta}_{E}$ when $\lambda=-2$. 


\subsubsection{Prior and posterior distribution}

The Bayesian estimation requires the choice of appropriate priors for the unknown parameters in addition to the experimental data. Here, it is assumed that the parameters follow the gamma $\left(a_{1}, b_{1}\right)$ and gamma $\left(a_{2}, b_{2}\right)$ prior distributions with $a_{1}>0, a_{2}>0, b_{1}>0$ and $b_{2}>0$. We also assumed that priors of $\alpha$ and $\beta$ are independent. So, the joint prior distribution of $\alpha$ and $\beta$ is obtained as

$$
\pi(\alpha, \beta) \propto \alpha^{a_{1}-1} \beta^{a_{2}-1} e^{-b_{1} \alpha-b_{2} \beta}, \quad \alpha>0, \beta>0 .
$$

Note that, when $a_{1}=a_{2}=b_{1}=b_{2}=0$, the prior distribution is the non-informative prior of $\alpha$ and $\beta$. Then, the joint density of the parameters and data is obtained as follows.

$$
L(\alpha, \beta \mid \mathbf{X}) \pi(\alpha, \beta) \propto \alpha^{s+a_{1}-1} \beta^{s+a_{2}-1} \prod_{i=1}^{s} x_{i: m: n}^{-(\alpha+1)} e^{-\beta x_{i: m: n}^{-\alpha}-b_{1} \alpha-b_{2} \beta}\left[1-e^{-\beta x_{i: m: n}^{-\alpha}}\right]^{R_{i}} \eta(\alpha, \beta),
$$

where $\mathbf{X}=\left(X_{1: m: n}, X_{2: m: n}, \ldots, X_{s: m: n}\right)$. Therefore, the posterior distribution of $\alpha$ and $\beta$, given data is obtained as

$$
\pi(\alpha, \beta \mid \mathbf{X}) \propto \frac{L(\alpha, \beta \mid \mathbf{X}) \pi(\alpha, \beta)}{\int_{0}^{\infty} \int_{0}^{\infty} L(\alpha, \beta \mid \mathbf{X}) \pi(\alpha, \beta) d \alpha d \beta}
$$

Now, we can obtain the Bayesian estimator of entropy under the SqL. It is obtained as

$$
\tilde{H}_{S}=\frac{\int_{0}^{\infty}[1+(1+1 / \alpha)(\gamma+\log \beta)-\log (\alpha \beta)] L(\alpha, \beta \mid \mathbf{X}) \pi(\alpha, \beta) d \alpha d \beta}{\int_{0}^{\infty} \int_{0}^{\infty} L(\alpha, \beta \mid \mathbf{X}) \pi(\alpha, \beta) d \alpha d \beta}
$$

\subsubsection{Lindley's approximation}

In this subsection, based on the GePH, we obtained several Bayesian estimator of entropy of the InW. These Bayesian estimator is derived against the SqL, PrL, GeL, and LiL. It is easily observed that all these estimates are in the form of ratio of two integrals for which simplified closed forms are not available. Thus we use the LiA to approximate all the Bayesian estimators. Nassar and Eissa (2004) noted that the LiA leads to

$$
\begin{aligned}
\hat{g}= & g\left(\hat{\theta}_{1}, \hat{\theta}_{2}\right)+\frac{1}{2}\left[\left(u_{11} \sigma_{11}+2 u_{12} \sigma_{12}+u_{22} \sigma_{22}\right)+l_{30}\left(u_{1} \sigma_{11}+u_{2} \sigma_{12}\right) \sigma_{11}+l_{03}\left(u_{2} \sigma_{22}+u_{1} \sigma_{21}\right) \sigma_{22}\right. \\
& \left.+l_{21}\left\{3 u_{1} \sigma_{11} \sigma_{12}+u_{2}\left(\sigma_{11} \sigma_{22}+2 \sigma_{12}^{2}\right)\right\}\right]+p_{1}\left(u_{1} \sigma_{11}+u_{2} \sigma_{21}\right)+p_{2}\left(u_{2} \sigma_{22}+u_{1} \sigma_{12}\right)
\end{aligned}
$$

where

$$
p=\log \pi\left(\theta_{1}, \theta_{2}\right), \quad p_{i}=\frac{\partial p}{\partial \theta_{i}}, \quad u_{i}=\frac{\partial g}{\partial \theta_{i}}, \quad \text { and } \quad u_{i j}=\frac{\partial^{2} g}{\partial \theta_{i} \theta_{j}}
$$

We further note that $l$ denotes the log-likelihood and $\sigma_{i j}$ denotes the $(i, j)^{\text {th }}$ element of the matrix $\left[-\partial^{2} l / \partial \theta_{1}^{i} \partial \theta_{2}^{j}\right]^{-1}$. Now, we compute $\hat{g}$ for our estimation problem with $\left(\theta_{1}, \theta_{2}\right)=(\alpha, \beta)$. Observe that 
for our problem we have

$$
\begin{aligned}
& l_{30}=\frac{\partial^{3} l}{\partial \alpha^{3}}=\eta_{\alpha^{3}}^{3}(\alpha, \beta)+\frac{2 s}{\alpha^{3}}+\beta \sum_{i=1}^{s} x_{i: m: n}^{-\alpha}\left(\log x_{i: m: n}\right)^{3}+\sum_{i=1}^{s} \frac{R_{i} x_{i: m: n}^{-\alpha}\left(\log x_{i: m: n}\right)^{3} e^{-\beta x_{i: m: n}^{-\alpha}}}{\left(1-e^{-\beta x_{i: m: n}^{-\alpha}}\right)^{3}} \\
& \times\left[e^{-\beta x_{i: m: n}^{-\alpha}}\left\{2-3 \beta x_{i: m: n}^{-\alpha}-e^{-\beta x_{i: m: n}^{-\alpha}}-\left(\beta x_{i: m: n}^{-\alpha}\right)^{2}\right\}+3 \beta x_{i: m: n}^{-\alpha}-\left(\beta x_{i: m: n}^{-\alpha}\right)-1\right], \\
& l_{03}=\frac{\partial^{3} l}{\partial \beta^{3}}=\eta_{\beta^{3}}^{3}(\alpha, \beta)+\frac{2 s}{\beta^{3}}+\sum_{i=1}^{s} R_{i} x_{i: m: n}^{-3 \alpha} e^{-\beta x_{i: m: n}^{-\alpha}} \frac{1+e^{-\beta x_{i: m: n}^{-\alpha}}}{\left(1-e^{-\beta x_{i: m: n}^{-\alpha}}\right)^{3}} \text {, } \\
& l_{21}=\frac{\partial^{3} l}{\partial \alpha^{2} \partial \beta}=\eta_{\alpha^{2} \beta}^{3}(\alpha, \beta)-\sum_{i=1}^{s} x_{i: m: n}^{-\alpha}\left(\log x_{i: m: n}\right)^{2}-\sum_{i=1}^{s} \frac{R_{i} x_{i: m: n}^{-\alpha}\left(\log x_{i: m: n}\right)^{2} e^{-\beta x_{i: m: n}^{-\alpha}}}{\left(1-e^{-\beta x_{i: m: n}^{-\alpha}}\right)^{3}} \\
& \times\left[e^{-\beta x_{i: m: n}^{-\alpha}}\left\{2-3 \beta x_{i: m: n}^{-\alpha}-e^{-\beta x_{i: m: n}^{-\alpha}}-\left(\beta x_{i: m: n}^{-\alpha}\right)^{2}\right\}+3 \beta x_{i: m: n}^{-\alpha}-\left(\beta x_{i: m: n}^{-\alpha}\right)-1\right] \text {, } \\
& l_{12}=\frac{\partial^{3} l}{\partial \alpha \partial \beta^{2}}=\eta_{\alpha \beta^{2}}^{3}(\alpha, \beta)+\sum_{i=1}^{s} \frac{R_{i} x_{i: m: n}^{-2 \alpha} \log x_{i: m: n} e^{-\beta x_{i: m: n}^{-\alpha}}}{\left(1-e^{-\beta x_{i: m: n}^{-\alpha}}\right)^{3}}\left[2-\beta x_{i: m: n}^{-\alpha}-2 e^{-\beta x_{i: m: n}^{-\alpha}}-\beta x_{i: m: n}^{-\alpha} e^{-\beta x_{i: m: n}^{-\alpha}}\right] \text {, }
\end{aligned}
$$

and

$$
p_{1}=\frac{a_{1}-1}{\alpha}-b_{1}, p_{2}=\frac{a_{2}-1}{\beta}-b_{2} .
$$

Here, for Case II,

$$
\begin{aligned}
& \eta_{\alpha^{3}}^{3}(\alpha, \beta)=\left[e^{-\beta T^{-\alpha}}\left\{2-3 \beta T^{-\alpha}-e^{-\beta T^{-\alpha}}-\left(\beta T^{-\alpha}\right)^{2}\right\}+3 \beta T^{-\alpha}-\left(\beta T^{-\alpha}\right)-1\right] \frac{R_{D+1}^{*} T^{-\alpha}(\log T)^{3} e^{-\beta T^{-\alpha}}}{\left(1-e^{-\beta T^{-\alpha}}\right)^{3}}, \\
& \eta_{\beta^{3}}^{3}(\alpha, \beta)=R_{D+1}^{*} T^{-3 \alpha} e^{-\beta T^{-\alpha}} \frac{1+e^{-\beta T^{-\alpha}}}{\left(1-e^{-\beta T^{-\alpha}}\right)^{3}}, \\
& \eta_{\alpha^{2} \beta}^{3}(\alpha, \beta)=-\left[e^{-\beta T^{-\alpha}}\left\{2-3 \beta T^{-\alpha}-e^{-\beta T^{-\alpha}}-\left(\beta T^{-\alpha}\right)^{2}\right\}+3 \beta T^{-\alpha}-\left(\beta T^{-\alpha}\right)-1\right] \frac{R_{D+1}^{*} T^{-\alpha}(\log T)^{2} e^{-\beta T^{-\alpha}}}{\left(1-e^{-\beta T^{-\alpha}}\right)^{3}}, \\
& \eta_{\alpha \beta^{2}}^{3}(\alpha, \beta)=\left[2-\beta T^{-\alpha}-2 e^{-\beta T^{-\alpha}}-\beta T^{-\alpha} e^{-\beta T^{-\alpha}}\right] \frac{R_{D+1}^{*} T^{-2 \alpha} \log T e^{-\beta T^{-\alpha}}}{\left(1-e^{-\beta T^{-\alpha}}\right)^{3}},
\end{aligned}
$$

for Case I and Case III,

$$
\eta_{\alpha^{3}}^{3}(\alpha, \beta)=\eta_{\beta^{3}}^{3}(\alpha, \beta)=\eta_{\alpha^{2} \beta}^{3}(\alpha, \beta)=\eta_{\alpha \beta^{2}}^{3}(\alpha, \beta)=0
$$

Also, we have

$$
\left(\begin{array}{ll}
\sigma_{11} & \sigma_{12} \\
\sigma_{21} & \sigma_{22}
\end{array}\right)=\left(\begin{array}{ll}
-l_{20} & -l_{11} \\
-l_{11} & -l_{02}
\end{array}\right)_{(\alpha, \beta)=(\hat{\alpha}, \hat{\beta})}^{-1},
$$


where

$$
\begin{aligned}
& l_{20}=\frac{\partial^{2} l(\alpha, \beta)}{\partial \alpha^{2}}=-\frac{s}{\alpha^{2}}-\beta \sum_{i=1}^{s}\left[R_{i} x_{i: m: n}^{-\alpha}\left(\log x_{i: m: n}\right)^{2} e^{-\beta x_{i: m: n}^{-\alpha}} \frac{\beta x_{i: m: n}^{-\alpha}+e^{-\beta x_{i: m: n}^{-\alpha}-1}}{\left(1-e^{-\beta x_{i: m: n}^{-\alpha}}\right)^{2}}+x_{i: m: n}^{-\alpha}\left(\log x_{i: m: n}\right)^{2}\right] \\
& +\eta_{\alpha^{2}}^{2}(\alpha, \beta), \\
& l_{02}=\frac{\partial^{2} l(\alpha, \beta)}{\partial \beta^{2}}=-\frac{s}{\beta^{2}}-\sum_{i=1}^{s} R_{i} \frac{x_{i: m: n}^{-2 \alpha} e^{-\beta x_{i: m: n}^{-\alpha}}}{\left(1-e^{-\beta x_{i: m: n}^{-\alpha}}\right)^{2}}+\eta_{\beta^{2}}^{2}(\alpha, \beta) \\
& l_{11}=\frac{\partial^{2} l(\alpha, \beta)}{\partial \alpha \partial \beta}=\sum_{i=1}^{s} R_{i} x_{i: m: n}^{-\alpha} \log x_{i: m: n} e^{-\beta x_{i: m: n}^{-\alpha}} \frac{\beta x_{i: m: n}^{-\alpha}+e^{-\beta x_{i: m: n}^{-\alpha}-1}}{\left(1-e^{-\beta x_{i: m: n}^{-\alpha}}\right)^{2}}+\sum_{i=1}^{s} x_{i: m: n}^{-\alpha} \log x_{i: m: n}+\eta_{\alpha \beta}^{2}(\alpha, \beta) .
\end{aligned}
$$

Here, for Case II,

$$
\begin{aligned}
& \eta_{\alpha^{2}}^{2}(\alpha, \beta)=-\beta R_{D+1}^{*} T^{-\alpha}(\log T)^{2} e^{-\beta T^{-\alpha}} \frac{\beta T^{-\alpha}+e^{-\beta T^{-\alpha}}-1}{\left[1-e^{-\beta T^{-\alpha}}\right]^{2}}, \\
& \eta_{\beta^{2}}^{2}(\alpha, \beta)=-R_{D+1}^{*} \frac{T^{-2 \alpha} e^{-\beta T^{-\alpha}}}{\left(1-e^{-\beta T^{-\alpha}}\right)^{2}}, \\
& \eta_{\alpha \beta}^{2}(\alpha, \beta)=R_{D+1}^{*} T^{-\alpha} \log T e^{-\beta T^{-\alpha}} \frac{\beta T^{-\alpha}+e^{-\beta T^{-\alpha}}-1}{\left[1-e^{-\beta T^{-\alpha}}\right]^{2}}
\end{aligned}
$$

for Case I and Case III,

$$
\eta_{\alpha}^{2}(\alpha, \beta)=\eta_{\beta}^{2}(\alpha, \beta)=\eta_{\alpha \beta}^{2}(\alpha, \beta)=0
$$

Now, we compute Bayesian estimate based on the LiA. First of all, we compute Bayesian estimator of entropy under the SqL. Now, we observe that

$$
\begin{aligned}
g(\alpha, \lambda) & =H(f)=1+\left(1+\frac{1}{\alpha}\right)(\gamma+\log \beta)-\log (\alpha \beta), \\
u_{1} & =-\frac{1}{\alpha^{2}}(\alpha+\gamma+\log \beta), \quad u_{2}=\frac{1}{\alpha \beta} \\
u_{11} & =\frac{1}{\alpha^{3}}(\alpha+2 \gamma+2 \log \beta), \quad u_{12}=u_{21}=-\frac{1}{\alpha^{2} \beta}, \quad u_{22}=-\frac{1}{\alpha \beta^{2}} .
\end{aligned}
$$

Utilizing expression in Equation (2.10), the approximate Bayesian estimator of entropy under the SqL is then obtained as

$$
\begin{aligned}
\hat{H}_{S}(f)= & \hat{H}(f)+\frac{1}{2}\left[\left(u_{11} \sigma_{11}+2 u_{12} \sigma_{12}+u_{22} \sigma_{22}\right)+l_{30}\left(u_{1} \sigma_{11}+u_{2} \sigma_{12}\right) \sigma_{11}+l_{03}\left(u_{2} \sigma_{22}+u_{1} \sigma_{21}\right) \sigma_{22}\right. \\
& \left.+l_{21}\left\{3 u_{1} \sigma_{11} \sigma_{12}+u_{2}\left(\sigma_{11} \sigma_{22}+2 \sigma_{12}^{2}\right)\right\}\right]+p_{1}\left(u_{1} \sigma_{11}+u_{2} \sigma_{21}\right)+p_{2}\left(u_{2} \sigma_{22}+u_{1} \sigma_{12}\right) .
\end{aligned}
$$


The case of the PrL is considered next. In the case of the PrL, we observe that,

$$
\begin{aligned}
g(\alpha, \lambda) & =H(f)^{2}, \quad u_{1}=-\frac{2}{\alpha^{2}}(\alpha+\gamma+\log \beta) H(f), \quad u_{2}=\frac{2}{\alpha \beta} H(f), \\
u_{11} & =-\frac{2}{\alpha^{4}}\left[-H(f)^{-1}(\alpha+\gamma+\log \beta)^{2}-\alpha(\alpha+2 \gamma+2 \log \beta)\right] H(f), \\
u_{12} & =u_{21}=\frac{2}{\alpha^{3} \beta}\left[-H(f)^{-1}(\alpha+\gamma+\log \beta)-\alpha\right] H(f), \quad u_{22}=-\frac{2}{\alpha^{2} \beta^{2}}\left[-H(f)^{-1}-\alpha\right] H(f) .
\end{aligned}
$$

Utilizing expression in Equation (2.10), the approximate Bayesian estimator of entropy under the PrL is then obtained as

$$
\begin{aligned}
\hat{H}_{P}(f)= & {\left[\hat{H}(f)^{2}+\frac{1}{2}\left[\left(u_{11} \sigma_{11}+2 u_{12} \sigma_{12}+u_{22} \sigma_{22}\right)+l_{30}\left(u_{1} \sigma_{11}+u_{2} \sigma_{12}\right) \sigma_{11}+l_{03}\left(u_{2} \sigma_{22}+u_{1} \sigma_{21}\right) \sigma_{22}\right.\right.} \\
& \left.\left.+l_{21}\left\{3 u_{1} \sigma_{11} \sigma_{12}+u_{2}\left(\tau_{11} \sigma_{22}+2 \sigma_{12}^{2}\right)\right\}\right]+p_{1}\left(u_{1} \sigma_{11}+u_{2} \sigma_{21}\right)+p_{2}\left(u_{2} \sigma_{22}+u_{1} \sigma_{12}\right)\right]^{\frac{1}{2}} .(2.13)
\end{aligned}
$$

The case of the GeL is considered next. In the case of the GeL, we observe that

$$
\begin{aligned}
g(\alpha, \lambda) & =H(f)^{-q}, \quad u_{1}=\frac{q}{\alpha^{2}}(\alpha+\gamma+\log \beta) H(f)^{-q-1}, \quad u_{2}=-\frac{q}{\alpha \beta} H(f)^{-q-1}, \\
u_{11} & =\frac{q}{\alpha^{4}}\left[(q+1) H(f)^{-1}(\alpha+\gamma+\log \beta)^{2}-\alpha(\alpha+2 \gamma+2 \log \beta)\right] H(f)^{-q-1}, \\
u_{12} & =u_{21}=-\frac{q}{\alpha^{3} \beta}\left[(q+1) H(f)^{-1}(\alpha+\gamma+\log \beta)-\alpha\right] H(f)^{-q-1}, \\
u_{22} & =\frac{q}{\alpha^{2} \beta^{2}}\left[(q+1) H(f)^{-1}-\alpha\right] H(f)^{-q-1} .
\end{aligned}
$$

Utilizing expression in Equation (2.10), the approximate Bayesian estimator of entropy under the GeL is then obtained as

$$
\begin{aligned}
\hat{H}_{E}(f)= & {\left[\hat{H}(f)^{-q}+\frac{1}{2}\left[\left(u_{11} \sigma_{11}+2 u_{12} \sigma_{12}+u_{22} \sigma_{22}\right)+l_{30}\left(u_{1} \sigma_{11}+u_{2} \sigma_{12}\right) \sigma_{11}+l_{03}\left(u_{2} \sigma_{22}+u_{1} \sigma_{21}\right) \sigma_{22}\right.\right.} \\
& \left.\left.+l_{21}\left\{3 u_{1} \sigma_{11} \sigma_{12}+u_{2}\left(\tau_{11} \sigma_{22}+2 \sigma_{12}^{2}\right)\right\}\right]+p_{1}\left(u_{1} \sigma_{11}+u_{2} \sigma_{21}\right)+p_{2}\left(u_{2} \sigma_{22}+u_{1} \sigma_{12}\right)\right]^{-\frac{1}{q}}
\end{aligned}
$$

Finally, the case of the LiL is considered. In the case of the LiL, we observe that

$$
\begin{aligned}
g(\alpha, \lambda) & =e^{-c H(f)}, \quad u_{1}=\frac{c}{\alpha^{2}}(\alpha+\gamma+\log \beta) e^{-c H(f)}, \quad u_{2}=-\frac{c}{\alpha \beta} e^{-c H(f)}, \\
u_{11} & =-\frac{c}{\alpha^{4}}\left[\alpha(\alpha+2 \gamma+2 \log \beta)-c(\alpha+\gamma+\log \beta)^{2}\right] e^{-c H(f)}, \\
u_{12} & =u_{21}=\frac{c}{\alpha^{3} \beta}[\alpha-c(\alpha+\gamma+\log \beta)] e^{-c H(f)}, \quad u_{22}=\frac{c}{\alpha^{3} \beta}(\alpha+c) e^{-c H(f)} .
\end{aligned}
$$

Utilizing expression in Equation (2.10), the approximate Bayesian estimator of entropy under LiL is then obtained as

$$
\begin{aligned}
\hat{H}_{L}(f)= & -\frac{1}{c} \log \left[e^{-c \hat{H}(f)}+\frac{1}{2}\left[\left(u_{11} \sigma_{11}+2 u_{12} \sigma_{12}+u_{22} \sigma_{22}\right)+l_{30}\left(u_{1} \sigma_{11}+u_{2} \sigma_{12}\right) \sigma_{11}+l_{03}\left(u_{2} \sigma_{22}+u_{1} \sigma_{21}\right) \sigma_{22}\right.\right. \\
& \left.\left.+l_{21}\left\{3 u_{1} \sigma_{11} \sigma_{12}+u_{2}\left(\sigma_{11} \sigma_{22}+2 \sigma_{12}^{2}\right)\right\}\right]+p_{1}\left(u_{1} \sigma_{11}+u_{2} \sigma_{21}\right)+p_{2}\left(u_{2} \sigma_{22}+u_{1} \sigma_{12}\right)\right] .
\end{aligned}
$$


Table 1: Estimates of entropy for example

\begin{tabular}{|c|c|c|c|c|c|c|c|c|c|c|}
\hline \multirow{2}{*}{ Case } & \multirow{2}{*}{$\hat{H}$} & \multirow{2}{*}{$\tilde{H}$} & \multirow{2}{*}{$\hat{H}_{S}$} & \multirow{2}{*}{$\hat{H}_{P}$} & \multicolumn{3}{|c|}{$\hat{H}_{E}$} & \multicolumn{3}{|c|}{$\hat{H}_{L}$} \\
\hline & & & & & $q=1.5$ & $q=1.8$ & $q=2.0$ & $c=1.5$ & $c=1.8$ & $c=2.0$ \\
\hline Case I & 2.5292 & 2.5349 & 2.5431 & 2.5597 & 2.5360 & 2.5340 & 2.5326 & 2.5173 & 2.5123 & 2.5090 \\
\hline Case II & 2.4264 & 2.4293 & 2.4370 & 2.4523 & 2.4315 & 2.4297 & 2.4285 & 2.4153 & 2.4111 & 2.4083 \\
\hline Case III & 2.2503 & 2.2441 & 2.2565 & 2.2719 & 2.2531 & 2.2515 & 2.2504 & 2.2384 & 2.2348 & 2.2325 \\
\hline
\end{tabular}

\section{Illustrative example and simulation results}

\subsection{Illustrative example}

We use the proposed estimators in Section 2 to analyze the real life data set. The real life data set are from the data on failure times of aircraft windshields (Blischke and Murthy, 2000). Blischke and Murthy (2000) examined the goodness-of-fit of the previous data to the InW graphically and found that InW fits the data very well. The ordered data are:

$0.301,0.309,0.557,0.943,1.070,1.124,1.248,1.281,1.281,1.303,1.432,1.480,1.505,1.506,1.568$, $1.615,1.619,1.652,1.652,1.757,1.795,1.866,1.876,1.899,1.911,1.912,1.914,1.981,2.010,2.038$, $2.085,2.089,2.097,2.135,2.154,2.190,2.194,2.223,2.224,2.229,2.300,2.324,2.349,2.385,2.481$, 2.610, 2.625, 2.632, 2.646, 2.661, 2.688, 2.823, 2.890, 2.902, 2.934, 2.962, 2.964, 3.000, 3.103, 3.114, $3.117,3.166,3.344,3.376,3.385,3.443,3.467,3.478,3.578,3.595,3.699,3.779,3.924,4.035,4.121$, $4.167,4.240,4.255,4.278,4.305,4.376,4.449,4.485,4.570,4.602,4.663,4.694$.

We now consider the case where the data set are progressively censored (PrC) with the following schemes: $m=77$ and $R_{77}=10, R_{1}=\cdots=R_{76}=0$. In this data set, we take Case I: $k=60$ and $T=3.00$, Case II: $k=60$ and $T=3.50$ and Case III: $k=60$ and $T=4.50$. The Bayesian estimators based on the non-informative prior are obtained. Furthermore, Bayesian estimates based on the GeL with $q=1.5,1.8$ and 2.0 are obtained along with the Bayesian estimates based on the LiL with $c=$ 1.5, 1.8 and 2.0. Table 1 presents the estimates of the entropy under the GePH.

In Table 1, we have tabulated entropy of the respective MLE and MPSE in the second and third column of the table. In the other columns, the Bayesian estimates of entropy are tabulated. We observed that Bayesian estimates of entropy using LiA obtained under LiL are marginally smaller than the corresponding MLE of entropy while opposite is true for the Bayesian estimate of entropy using LiA obtained under the SqL, PrL and GeL.

\subsection{Simulation results}

We use Monte Carlo simulations to compare different methods since the performance of the different methods cannot be compared theoretically. We consider different $n, m, k$, and $T$. We have used three different PrC, namely; Scheme (Sch.) I $\left(R_{m}=n-m\right.$ and $R_{i}=0$ for $\left.i \neq m\right)$, Sch. II $\left(R_{m / 2}=n-m\right.$ and $R_{i}=0$ for $\left.i \neq m / 2\right)$ and Sch. III $\left(R_{1}=R_{m}=(n-m) / 2\right.$ and $R_{i}=0$ for $\left.i \neq 1, m\right)$. Without loss of generality, we take $\alpha=\beta=2$ in each case. We replicate the process 1,000 times for each case. The associated MLE and MPSE of entropy are computed using a Newton-Raphson method. In Bayesian estimation, hyperparameters take values of $a_{1}=a_{2}=b_{1}=b_{2}=0.0001$. Bayesian estimates of entropy are derived with respect to four different loss functions - SqL, PLF, GeL, and LiL. The LiA and TiK (Lee, 2017) have then been used to derive approximate expressions for these estimates. Under GeL associated estimators are obtained for $q=1.5,1.8$, and 2.0. Also, under LiL associated estimates are obtained for $c=1.5,1.8$ and 2.0. Finally, different schemes have been taken into consideration to compute root mean squared error (RMSE) and bias values of all estimates. These 
values are also tabulated in Table 2. We present the following discussions based on the RMSEs and biases.

In Table 2, RMSE and bias values of all estimates of entropy are presented for various choices of $n, m$ and PrC. We have tabulated entropy of the respective MLE and MPSE in the fifth and sixth column of the table. In the other columns, the Bayesian estimates of entropy using LiA and TiK are tabulated. All columns uniformly contain four values. The first value corresponds to the RMSE of entropy using the LiA and the second value represents the corresponding bias. The third and fourth values, respectively, correspond to the RMSE and bias of entropy using TiK.

The RMSE and bias decrease as sample size $n$ increases. For fixed sample size $n$, the RMSE and bias decrease generally as the number of $\operatorname{PrC}$ samples $R_{i}$ decreases. For Fixed sample $n$ and $\operatorname{PrC}$ data size $m$, the RMSE and bias decrease generally as the time $T$ increases. For fixed time $T$, sample and $\operatorname{PrC}$ data size, the RMSE and bias decrease generally as the number of guarantee sample size $k$ increases. It is also observed that the MLE for schemes I and II behave quite similarly in terms of RMSE and bias. The MLE for scheme III has smaller RMSE and bias than the corresponding MLE for the two other schemes. We also observed that Bayesian estimators are superior to the respective MLE and MPSE in terms of RMSE values. In addition, we observed that Bayesian estimators using $\mathrm{LiA}$ are superior to respective Bayesian estimators using TiK (Lee, 2017) in terms of RMSE values. In particular, respective Bayesian estimators of entropy under LiL are better than the corresponding MLE and MPSE. For Bayesian estimating the entropy under LiL, the choice $c=1.5$ seems reasonable for LiA.

\section{Conclusions}

In this paper, we consider the Bayesian estimators, MLE and MPSE of the entropy of the InW based on the GePH. The MLE of entropy is superior to respective MPSE in terms of the RMSE and bias. We further consider the Bayesian estimation of the entropy based on several priors - SqL, PrL, GeL, and LiL. It is observed that Bayesian estimators cannot be derived in closed form, and we use the LiA for the Bayesian estimators. The Bayesian estimators of entropy are superior to the respective MPSE and MLE in terms of MSEs. The Bayesian estimators of entropy using LiA are superior to respective Bayesian estimators of entropy using TiK in terms of MSEs. The choice of LiL is a reasonable choice for the Bayesian estimation of entropy. For Bayesian estimating the entropy under LiL, the choice $c=1.5$ is a reasonable. We focused on the entropy estimate of the $\mathrm{InW}$ based on the $\mathrm{GePH}$; however, the estimation of the entropy from other distributions based on the $\mathrm{GePH}$ is also of potential interest in future research.

\section{Acknowledgements}

This research was supported by Daegu University Research Grant, 2018. 
Table 2: The relative RMSEs and biases of entropy estimators with MLE, MPSE and Bayesian estimator

\begin{tabular}{|c|c|c|c|c|c|c|c|c|c|c|c|c|}
\hline & & & & & & & $T=2.0$ & & & & & \\
\hline$n$ & $m k$ & Sch. & $\hat{H}$ & $\tilde{H}$ & $\hat{H}_{S}$ & $\hat{H}_{P}$ & & $\hat{H}_{E}$ & & & $\hat{H}_{L}$ & \\
\hline & & & & & & & $q=1.5$ & $q=1.8$ & $q=2.0$ & $c=1.5$ & $c=1.8$ & $c=2.0$ \\
\hline & & & 0.3963 & 0.4267 & 0.4166 & 0.4319 & 0.4202 & 0.4210 & 0.4216 & 0.3890 & 0.3881 & 0.3883 \\
\hline & & $\mathrm{I}$ & $(-0.0683)$ & (0.0173) & $(0.0083)$ & (0.0759) & $(-0.0813)$ & $(-0.0947)$ & ) $(-0.1033)$ & $(-0.0960)$ & $(-0.1153)$ & $(-0.1274)$ \\
\hline & & 1 & & & 0.4273 & 0.4275 & 0.4244 & 0.4221 & 0.4219 & 0.4241 & 0.4219 & 0.4205 \\
\hline & & & & & $(0.0186)$ & (0.0206) & (0.0043) & $(0.0040)$ & $(0.0029)$ & $(0.0450)$ & $(0.0414)$ & $(0.0390)$ \\
\hline & & & 0.4028 & 0.4413 & 0.4290 & 0.4476 & 0.4272 & 0.4273 & 0.4276 & 0.3935 & 0.3918 & 0.3917 \\
\hline & & U & $(-0.0616)$ & $(0.0259)$ & $(0.0163)$ & $(0.0874)$ & $(-0.0775)$ & $(-0.0915)$ & $(-0.1005)$ & $(-0.0944)$ & $(-0.1146)$ & $(-0.1272)$ \\
\hline & 0 & 11 & & & 0.4421 & 0.4427 & 0.4382 & 0.4357 & 0.4354 & 0.4390 & 0.4363 & 0.4346 \\
\hline & & & & & $(0.0268)$ & $(0.0287)$ & (0.0115) & $(0.0111)$ & (0.0099) & $(0.0537)$ & $(0.0498)$ & $(0.0472)$ \\
\hline & & & 0.3899 & 0.4232 & 0.4137 & 0.4314 & 0.4125 & 0.4128 & 0.4130 & 0.3817 & 0.3802 & 0.3801 \\
\hline & & UU & $(-0.0596)$ & $(0.0235)$ & $(0.0152)$ & $(0.0835)$ & $(-0.0732)$ & $(-0.0865)$ & ) $(-0.0951)$ & $(-0.0899)$ & $(-0.1092)$ & $(-0.1213)$ \\
\hline & & 1111 & & & 0.4258 & 0.4262 & 0.4220 & 0.4216 & 0.4214 & 0.4234 & 0.4210 & 0.4195 \\
\hline & & & & & $(0.0253)$ & $(0.0270)$ & (0.0110) & $(0.0093)$ & $(0.0082)$ & $(0.0511)$ & $(0.0475)$ & $(0.0451)$ \\
\hline & & & 0.3895 & 0.4138 & 0.4061 & 0.4199 & 0.4127 & 0.4139 & 0.4148 & 0.3836 & 0.3833 & 0.3840 \\
\hline & & & $(-0.0715)$ & $(0.0142)$ & $(0.0041)$ & $(0.0714)$ & $(-0.0848)$ & $(-0.0980)$ & $(-0.1066)$ & $(-0.0988)$ & $(-0.1178)$ & $(-0.1298)$ \\
\hline & & 1 & & & 0.4142 & 0.4142 & 0.4122 & 0.4099 & 0.4097 & 0.4114 & 0.4095 & 0.4083 \\
\hline & & & & & $(0.0136)$ & (0.0155) & $(-0.0005)$ & $(-0.0008)$ & $(-0.0019)$ & $(0.0402)$ & $(0.0367)$ & $(0.0344)$ \\
\hline & & & 0.3880 & 0.4162 & 0.4065 & 0.4223 & 0.4113 & 0.4124 & 0.4132 & 0.3821 & 0.3818 & 0.3825 \\
\hline & & & $(-0.0677)$ & $(0.0157)$ & $(0.0078)$ & $(0.0782)$ & $(-0.0843)$ & $(-0.0980)$ & $(-0.1068)$ & $(-0.0997)$ & $(-0.1194)$ & $(-0.1317)$ \\
\hline & 8 & 11 & & & 0.4156 & 0.4156 & 0.4133 & 0.4109 & 0.4107 & 0.4133 & 0.4112 & 0.4099 \\
\hline & & & & & (0.0168) & $(0.0185)$ & $(0.0020)$ & (0.0016) & $(0.0005)$ & $(0.0441)$ & $(0.0404)$ & $(0.0379)$ \\
\hline & & & 0.3823 & 0.4102 & 0.4017 & 0.4178 & 0.4042 & 0.4049 & 0.4056 & 0.3758 & 0.3751 & 0.3754 \\
\hline & & II & $(-0.0629)$ & $(0.0182)$ & (0.0104) & $(0.0784)$ & $(-0.0769)$ & $(-0.0901)$ & $(-0.0986)$ & $(-0.0927)$ & $(-0.1117)$ & $(-0.1237)$ \\
\hline & & IIII & & & 0.4109 & 0.4110 & 0.4081 & 0.4079 & 0.4077 & 0.4091 & 0.4070 & 0.4057 \\
\hline & & & & & (0.0196) & (0.0213) & (0.0057) & (0.0040) & $(0.0029)$ & $(0.0457)$ & $(0.0422)$ & (0.0399) \\
\hline & & & 0.4347 & 0.4532 & 0.4445 & 0.4497 & 0.4681 & 0.4701 & 0.4719 & 0.4295 & 0.4316 & 0.4338 \\
\hline & & & $(-0.1240)$ & $(-0.0113)$ & $(-0.0304)$ & $(0.0465)$ & $(-0.1416)$ & $(-0.1583)$ & $(-0.1683)$ & $(-0.1496)$ & $(-0.1717)$ & $(-0.1855)$ \\
\hline & & 1 & & & 0.4554 & 0.4550 & 0.4553 & 0.4536 & 0.4518 & 0.4474 & 0.4453 & 0.4439 \\
\hline & & & & & $(-0.0133)$ & $(-0.0100)$ & $(-0.0312)$ & $(-0.0321)$ & $(-0.0322)$ & $(0.0188)$ & $(0.0144)$ & (0.0116) \\
\hline & & & 0.4289 & 0.4809 & 0.4526 & 0.4676 & 0.4752 & 0.4765 & 0.4594 & 0.4342 & 0.4345 & 0.4219 \\
\hline & 6 & $\mathrm{JI}$ & $(-0.0848)$ & $(0.0124)$ & $(-0.0006)$ & $(0.0767)$ & $(-0.1177)$ & $(-0.1344)$ & $(-0.1307)$ & $(-0.1321)$ & $(-0.1560)$ & $(-0.1565)$ \\
\hline & 0 & 11 & & & 0.4815 & 0.4818 & 0.4787 & 0.4748 & 0.4747 & 0.4752 & 0.4721 & 0.4702 \\
\hline & & & & & $(0.0131)$ & $(0.0157)$ & $(-0.0069)$ & $(-0.0065)$ & $(-0.0080)$ & $(0.0460)$ & $(0.0409)$ & $(0.0376)$ \\
\hline & & & 0.4087 & 0.4454 & 0.4334 & 0.4510 & 0.4353 & 0.4360 & 0.4365 & 0.4003 & 0.3993 & 0.3997 \\
\hline & & 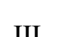 & $(-0.0732)$ & $(0.0162)$ & $(0.0083)$ & $(0.0822)$ & $(-0.0908)$ & $(-0.1054)$ & $(-0.1148)$ & $(-0.1069)$ & $(-0.1279)$ & $(-0.1410)$ \\
\hline & & & & & 0.4471 & 0.4476 & 0.4434 & 0.4411 & 0.4408 & 0.4429 & 0.4402 & 0.4384 \\
\hline & & & & & (0.0194) & $(0.0215)$ & $(0.0032)$ & $(0.0027)$ & (0.0014) & $(0.0477)$ & $(0.0435)$ & $(0.0408)$ \\
\hline & & & 0.4255 & 0.4401 & 0.4312 & 0.4373 & 0.4584 & 0.4618 & 0.4641 & 0.4221 & 0.4250 & 0.4276 \\
\hline & & & & > & $(-0.0306)$ & (0.0437) & $(-0.1430)$ & $(-0.1583)$ & $(-0.1681)$ & $(-0.1486)$ & $(-0.1704)$ & $(-0.1841)$ \\
\hline & & 1 & & & 0.4407 & 0.4400 & 0.4418 & 0.4401 & 0.4384 & 0.4326 & 0.4309 & 0.4299 \\
\hline & & & & & $(-0.0209)$ & $(-0.0178)$ & $(-0.0384)$ & $(-0.0392)$ & $(-0.0393)$ & (0.0114) & $(0.0072)$ & $(0.0045)$ \\
\hline & & & 0.4099 & 0.4281 & 0.4206 & 0.4300 & 0.4505 & 0.4541 & 0.4414 & 0.4165 & 0.4202 & 0.4106 \\
\hline & & & $(-0.0960)$ & $(-0.0224)$ & $(-0.0177)$ & $(0.0582)$ & $(-0.1410)$ & $(-0.1564)$ & $(-0.1423)$ & $(-0.1512)$ & $(-0.1729)$ & $(-0.1648)$ \\
\hline & 8 & II & & & 0.4298 & 0.4288 & 0.4316 & 0.4277 & 0.4279 & 0.4217 & 0.4202 & 0.4193 \\
\hline & & & & & $(-0.0248)$ & $(-0.0229)$ & $(-0.0427)$ & $(-0.0422)$ & $(-0.0436)$ & $(0.0083)$ & $(0.0040)$ & $(0.0013)$ \\
\hline & & & 0.3890 & 0.4102 & 0.4023 & 0.4152 & 0.4142 & 0.4162 & 0.4176 & 0.3848 & 0.3860 & 0.3875 \\
\hline & & III & $(-0.0830)$ & $(0.0027)$ & $(-0.0058)$ & $(0.0669)$ & $(-0.1017)$ & $(-0.1159)$ & $(-0.1250)$ & $(-0.1156)$ & $(-0.1357)$ & $(-0.1483)$ \\
\hline & & 111 & & & 0.4105 & 0.4102 & 0.4092 & 0.4080 & 0.4069 & 0.4097 & 0.4089 & 0.4037 \\
\hline & & & & & $(0.0031)$ & (0.0048) & $(-0.0124)$ & $(-0.0128)$ & $(-0.0141)$ & $(0.0317)$ & $(0.0279)$ & $(0.0254)$ \\
\hline
\end{tabular}




\begin{tabular}{|c|c|c|c|c|c|c|c|c|c|c|c|}
\hline \multicolumn{12}{|c|}{ Continued } \\
\hline & & & & & & $T=2.0$ & & & & & \\
\hline & & $\hat{H}$ & $\tilde{U}$ & & $\hat{H}$ & & $\hat{H}_{E}$ & & & $\hat{H}_{L}$ & \\
\hline$m k$ & Sch. & $H$ & $H$ & $H_{S}$ & $H_{P}$ & $q=1.5$ & $q=1.8$ & $q=2.0$ & $c=1.5$ & $c=1.8$ & $c=2.0$ \\
\hline & & 0.3084 & 0.3192 & 0.3159 & 0.3229 & 0.3212 & 0.3224 & 0.3232 & 0.3063 & 0.3065 & 0.3070 \\
\hline & & $(-0.0561)$ & $(-0.0054)$ & $(-0.0061)$ & $(0.0395)$ & $(-0.0648)$ & $(-0.0738)$ & $(-0.0796)$ & $(-0.0741)$ & $(-0.0871)$ & $(-0.0954)$ \\
\hline & 1 & & & 0.3202 & 0.3202 & 0.3197 & 0.3196 & 0.3196 & 0.3202 & 0.3195 & 0.3191 \\
\hline & & & & $(-0.0034)$ & $(-0.0026)$ & $(-0.0093)$ & $(-0.0100)$ & $(-0.0105)$ & $(0.0190)$ & $(0.0175)$ & $(0.0165)$ \\
\hline & & 0.3084 & 0.3201 & 0.3180 & 0.3269 & 0.3214 & 0.3165 & 0.3175 & 0.3060 & 0.3060 & 0.3065 \\
\hline & & $(-0.0503)$ & $(-0.0009)$ & $(-0.0006)$ & $(0.0480)$ & $(-0.0620)$ & $(-0.0758)$ & $(-0.0817)$ & $(-0.0727)$ & $(-0.0863)$ & $(-0.0951)$ \\
\hline 12 & 11 & & & 0.3217 & 0.3217 & 0.3209 & 0.3209 & 0.3208 & 0.3223 & 0.3215 & 0.3211 \\
\hline & & & & $(0.0009)$ & $(0.0015)$ & $(-0.0054)$ & $(-0.0061)$ & $(-0.0066)$ & $(0.0243)$ & $(0.0227)$ & $(0.0216)$ \\
\hline & & 0.3044 & 0.3176 & 0.3146 & 0.3234 & 0.3164 & 0.3171 & 0.3177 & 0.3018 & 0.3014 & 0.3016 \\
\hline & & $(-0.0468)$ & $(0.0032)$ & (0.0008) & $(0.0469)$ & $(-0.0563)$ & $(-0.0651)$ & $(-0.0709)$ & $(-0.0671)$ & $(-0.0800)$ & $(-0.0883)$ \\
\hline & III & & & 0.3184 & 0.3184 & 0.3175 & 0.3174 & 0.3174 & 0.3192 & 0.3186 & 0.3181 \\
\hline & & & & $(0.0026)$ & $(0.0033)$ & $(-0.0032)$ & $(-0.0039)$ & $(-0.0044)$ & $(0.0246)$ & $(0.0231)$ & $(0.0221)$ \\
\hline 26 & & 0.3047 & 0.3127 & 0.3102 & 0.3163 & 0.3172 & 0.3185 & 0.3195 & 0.3029 & 0.3035 & 0.3042 \\
\hline & & $(-0.0576)$ & $(-0.0093)$ & $(-0.0086)$ & $(0.0368)$ & $(-0.0667)$ & $(-0.0755)$ & $(-0.0813)$ & $(-0.0757)$ & $(-0.0885)$ & $(-0.0967)$ \\
\hline & 1 & & & 0.3134 & 0.3133 & 0.3131 & 0.3131 & 0.3130 & 0.3128 & 0.3123 & 0.3120 \\
\hline & & & & $(-0.0073)$ & $(-0.0065)$ & $(-0.0131)$ & $(-0.0138)$ & $(-0.0142)$ & $(0.0150)$ & $(0.0135)$ & $(0.0125)$ \\
\hline & & 0.3022 & 0.3083 & 0.3073 & 0.3140 & 0.3150 & 0.3693 & 0.3706 & 0.3008 & 0.3016 & 0.3026 \\
\hline & & $(-0.0547)$ & $(-0.0096)$ & $(-0.0075)$ & $(0.0403)$ & $(-0.0667)$ & $(-0.0876)$ & $(-0.0946)$ & $(-0.0768)$ & $(-0.0899)$ & $(-0.0984)$ \\
\hline 14 & 11 & & & 0.3103 & 0.3101 & 0.3100 & 0.3100 & 0.3100 & 0.3096 & 0.3091 & 0.3088 \\
\hline & & & & $(-0.0066)$ & $(-0.0060)$ & $(-0.0127)$ & $(-0.0134)$ & $(-0.0139)$ & $(0.0163)$ & $(0.0148)$ & $(0.0138)$ \\
\hline & & 0.2996 & 0.3073 & 0.3068 & 0.3143 & 0.3114 & 0.3124 & 0.3132 & 0.2977 & 0.2979 & 0.2984 \\
\hline & & $(-0.0498)$ & $(-0.0062)$ & $(-0.0035)$ & $(0.0422)$ & $(-0.0595)$ & $(-0.0681)$ & $(-0.0738)$ & $(-0.0699)$ & $(-0.0825)$ & $(-0.0906)$ \\
\hline & III & & & 0.3099 & 0.3098 & 0.3093 & 0.3093 & 0.3093 & 0.3100 & 0.3095 & 0.3091 \\
\hline & & & & $(-0.0022)$ & $(-0.0012)$ & $(-0.0079)$ & $(-0.0086)$ & $(-0.0090)$ & (0.0196) & $(0.0182)$ & (0.0172) \\
\hline 30 & & 0.3500 & 0.3617 & 0.3579 & 0.3652 & 0.3684 & 0.3700 & 0.3712 & 0.3469 & 0.3474 & 0.3483 \\
\hline & I & $(-0.0606)$ & $(0.0023)$ & $(0.0029)$ & $(0.0547)$ & $(-0.0741)$ & $(-0.0849)$ & $(-0.0919)$ & $(-0.0805)$ & $(-0.0963)$ & $(-0.1063)$ \\
\hline & & & & 0.3625 & 0.3623 & 0.3594 & 0.3594 & 0.3594 & 0.3611 & 0.3604 & 0.3599 \\
\hline & & & & $(0.0033)$ & $(0.0046)$ & $(-0.0027)$ & $(-0.0036)$ & $(-0.0041)$ & $(0.0308)$ & $(0.0289)$ & $(0.0276)$ \\
\hline & & 0.3493 & 0.3569 & 0.3544 & 0.3623 & 0.3673 & 0.3693 & 0.3706 & 0.3467 & 0.3480 & 0.3492 \\
\hline & & $(-0.0556)$ & $(-0.0067)$ & $(-0.0028)$ & $(0.0543)$ & $(-0.0767)$ & $(-0.0876)$ & $(-0.0946)$ & $(-0.0871)$ & $(-0.1027)$ & $(-0.1126)$ \\
\hline 12 & II & & & 0.3583 & 0.3579 & 0.3557 & 0.3557 & 0.3558 & 0.3561 & 0.3555 & 0.3551 \\
\hline & & & & $(-0.0047)$ & $(-0.0042)$ & $(-0.0109)$ & $(-0.0118)$ & $(-0.0125)$ & $(0.0232)$ & $(0.0213)$ & $(0.0200)$ \\
\hline & & 0.3280 & 0.3419 & 0.3398 & 0.3505 & 0.3419 & 0.3425 & 0.3429 & 0.3241 & 0.3234 & 0.3235 \\
\hline & 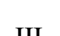 & $(-0.0366)$ & $(0.0142)$ & (0.0144) & $(0.0645)$ & $(-0.0492)$ & $(-0.0588)$ & $(-0.0651)$ & $(-0.0607)$ & $(-0.0748)$ & $(-0.0838)$ \\
\hline & & & & 0.3435 & 0.3434 & 0.3397 & 0.3396 & 0.3396 & 0.3444 & 0.3437 & 0.3431 \\
\hline & & & & $(0.0150)$ & $(0.0157)$ & (0.0099) & $(0.0092)$ & $(0.0086)$ & $(0.0391)$ & $(0.0374)$ & $(0.036$ \\
\hline & & 0.3391 & 0.3416 & 0.3392 & 0.3423 & 0.3569 & 0.3594 & 0.3611 & 0.3373 & 0.3393 & 0.3409 \\
\hline & & $(-0.0723)$ & $(-0.0157)$ & $(-0.0145)$ & $(0.0356)$ & $(-0.0867)$ & $(-0.0970)$ & $(-0.1036)$ & $(-0.0920)$ & $(-0.1068)$ & $(-0.1162)$ \\
\hline & I & & & 0.3429 & 0.3424 & 0.3405 & 0.3406 & 0.3406 & 0.3392 & 0.3388 & 0.3385 \\
\hline & & & & $(-0.0150)$ & $(-0.0138)$ & $(-0.0205)$ & $(-0.0213)$ & $(-0.0219)$ & $(0.0115)$ & $(0.0097)$ & $(0.0086)$ \\
\hline & & 0.3394 & 0.3418 & 0.3383 & 0.3416 & 0.3571 & 0.3596 & 0.3613 & 0.3385 & 0.3408 & 0.3425 \\
\hline & & $(-0.0644)$ & $(-0.0232)$ & $(-0.0205)$ & $(0.0338)$ & $(-0.0862)$ & $(-0.0960)$ & $(-0.1024)$ & $(-0.0960)$ & $(-0.1101)$ & $(-0.1190)$ \\
\hline 14 & II & & & 0.3424 & 0.3418 & 0.3405 & 0.3406 & 0.3407 & 0.3379 & 0.3376 & 0.3374 \\
\hline & & & & $(-0.0226)$ & $(-0.0223)$ & $(-0.0280)$ & $(-0.0289)$ & $(-0.0294)$ & $(0.0033)$ & $(0.0017)$ & $(0.0006)$ \\
\hline & & 0.3151 & 0.3227 & 0.3206 & 0.3280 & 0.3286 & 0.3300 & 0.3309 & 0.3129 & 0.3135 & 0.3143 \\
\hline & & $(-0.0469)$ & $(0.0012)$ & $(0.0007)$ & $(0.0497)$ & $(-0.0601)$ & $(-0.0693)$ & $(-0.0754)$ & $(-0.0705)$ & $(-0.0840)$ & $(-0.0926)$ \\
\hline & 1111 & & & 0.3233 & 0.3230 & 0.3200 & 0.3200 & 0.3200 & 0.3226 & 0.3220 & 0.3217 \\
\hline & & & & $(0.0006)$ & $(0.0011)$ & $(-0.0042)$ & $(-0.0049)$ & $(-0.0054)$ & $(0.0241)$ & $(0.0226)$ & $(0.0215)$ \\
\hline
\end{tabular}




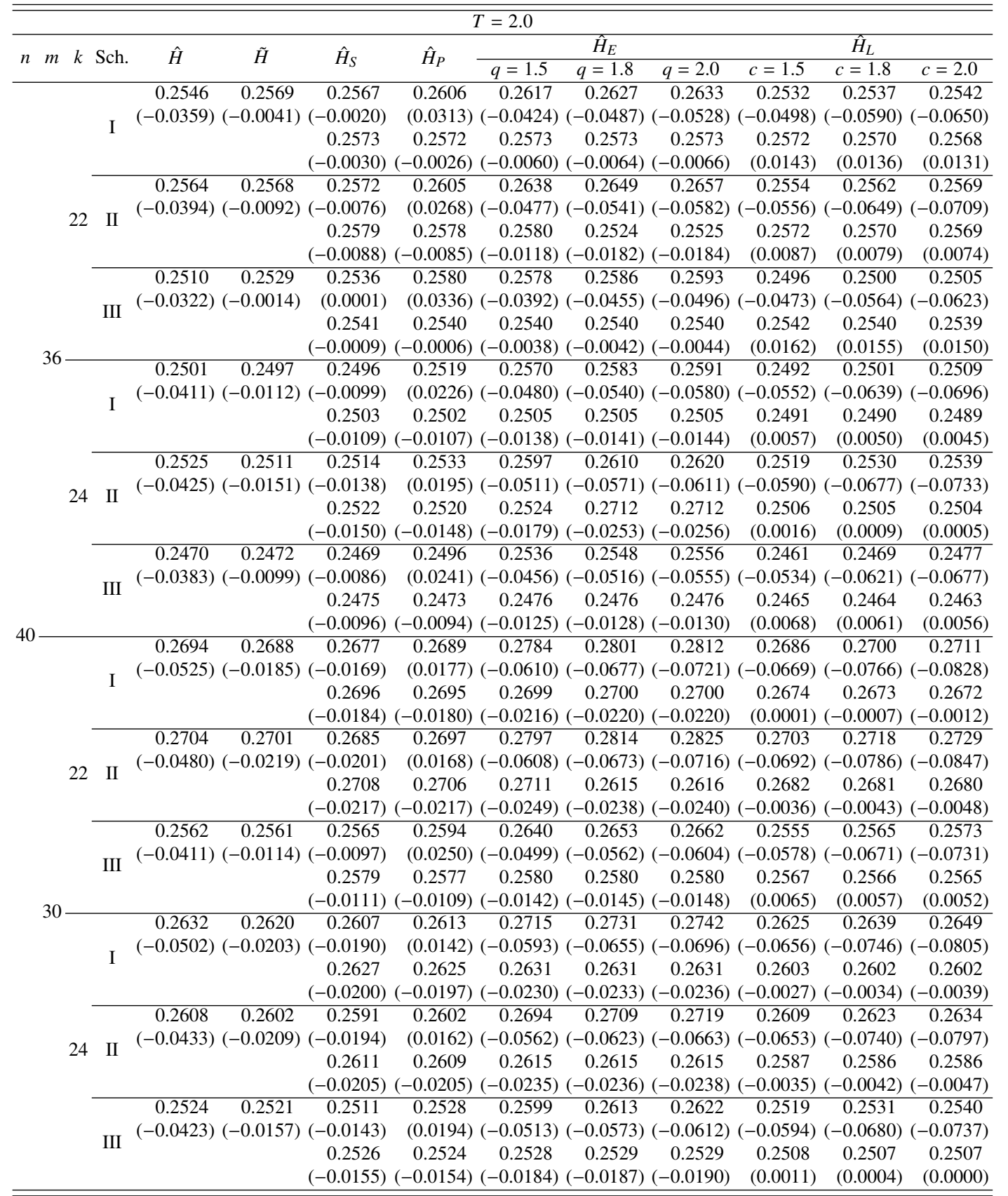




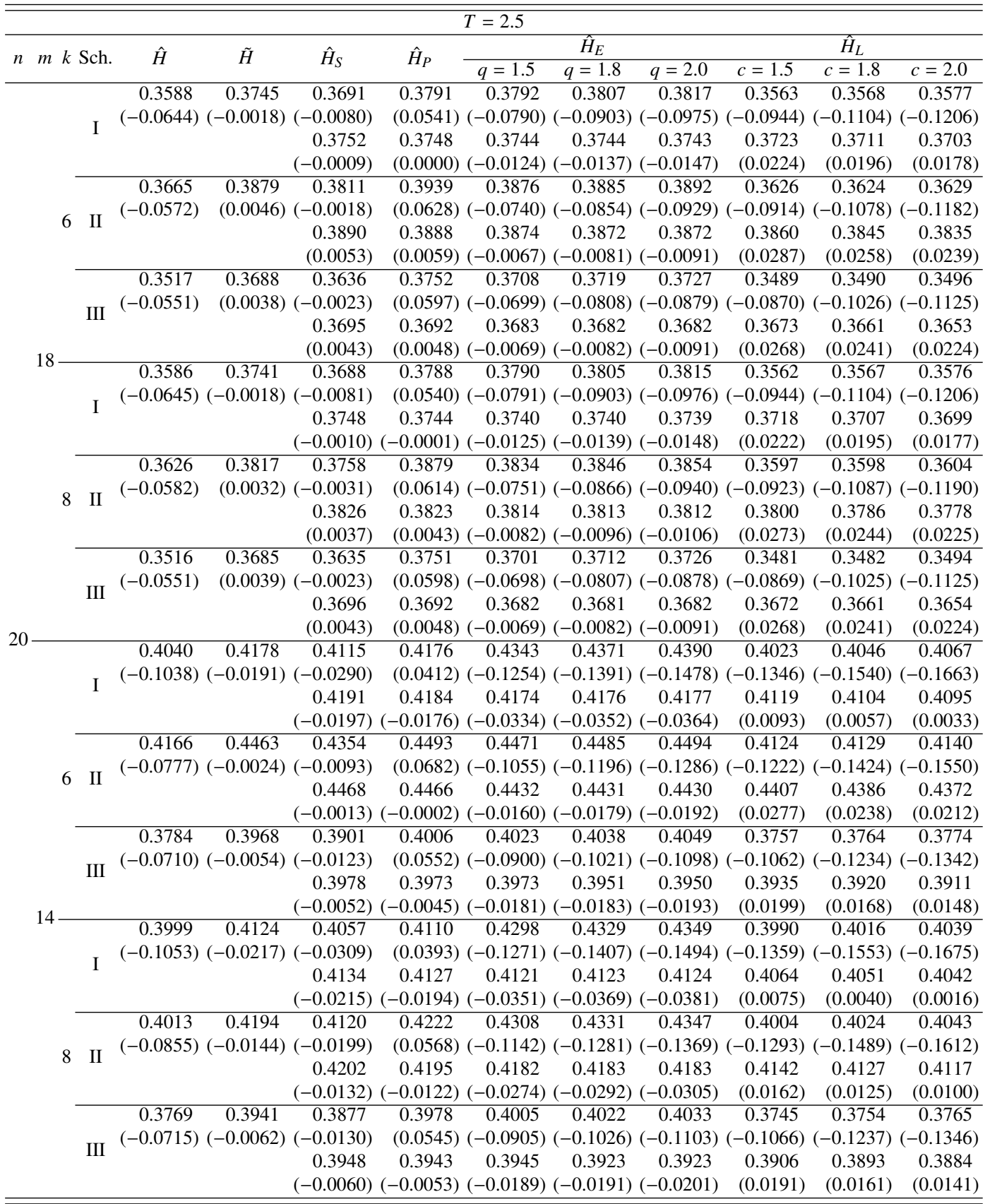




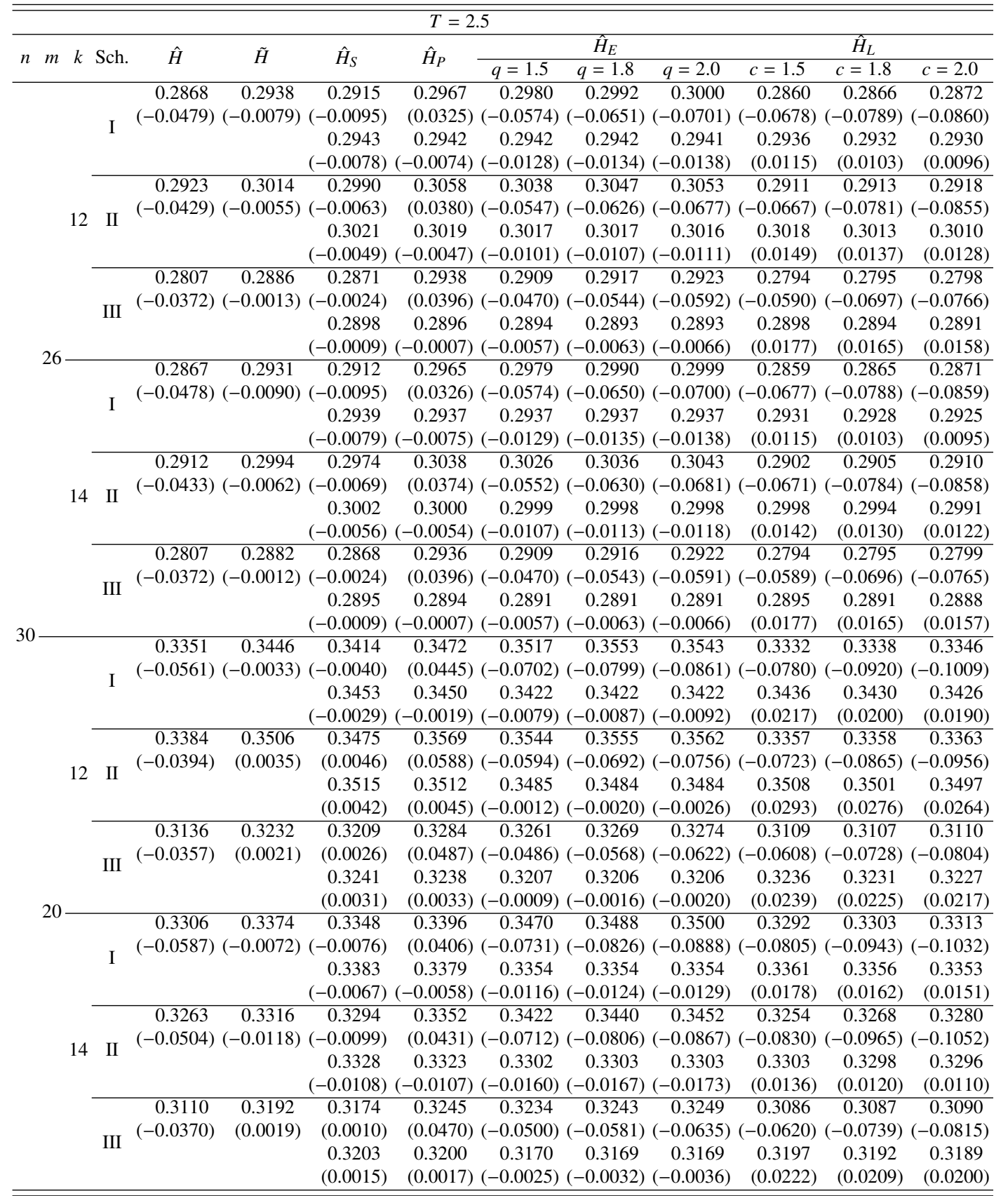




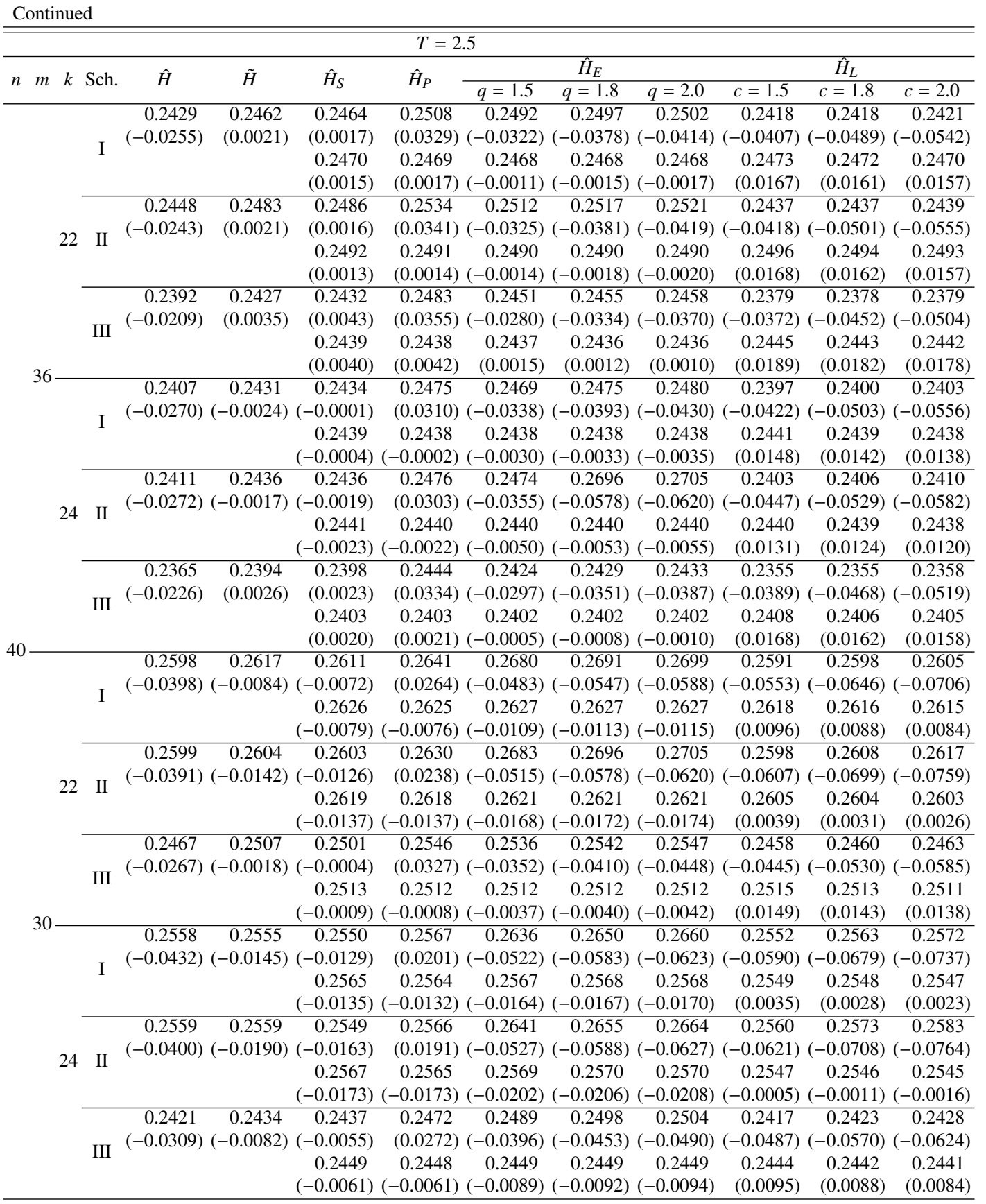




\section{References}

Baratpour S, Ahmadi J, and Arghami NR (2007). Entropy properties of record statistics, Statistical Papers, 48, 197-213.

Basu AP and Ebrahimi N (1991). Bayesian approach to life testing and reliability estimation using asymmetric loss function, Journal of Statistical Planning and Inference, 29, 21-31.

Blischke WR and Murthy DNP (2000). Reliability : Modeling, Prediction, and Optimization, Wiley, New York.

Calabria R and Pulcini G (1990). On the maximum likelihood and lease squares estimation in inverse Weibull distribution, Statistics Applicata, 2, 53-66.

Calabria R and Pulcini G (1994). Bayes 2-sample prediction for the inverse Weibull distribution, Communications in Statistics - Theory and Methods, 23, 1811-1824.

Cheng RCH and Amin NAK (1983). Estimating parameters in continuous univariate distributions with a shifted origin, Journal of the Royal Statistical Society B, 45, 394-403.

Cho Y, Sun H, and Lee K (2014). An estimation of the entropy for a Rayleigh distribution based on doubly-generalized type-II hybrid censored samples, Entropy, 16, 3655-3669.

Cho Y, Sun H, and Lee K (2015a). Estimating the entropy of a Weibull distribution under generalized progressive hybrid censoring, Entropy, 17, 101-122.

Cho Y, Sun H, and Lee K (2015b). Exact likelihood inference for an exponential parameter under generalized progressive hybrid censoring scheme, Statistical Methodology, 23, 18-34.

Cover TM and Thomas JA (2005). Elements of Information Theory, Wiley, Hoboken, NJ.

Huzurbazar VS (1948). The likelihood equation, consistency and the maxima of the likelihood function, Annals of Eugenics, 14, 185-200.

Kang S, Cho Y, Han J, and Kim J (2012). An estimation of the entropy for a double exponential distribution based on multiply type-II censored samples, Entropy, 14, 161-173.

Keller AZ, Giblin MT, and Farnworth NR (1985). Reliability analysis of commercial vehicle engines, Reliability Engineering, 10, 89-102.

Khan MS, Pasha GR, and Pasha AH (2008). Theoretical analysis of inverse Weibull distribution, WSEAS Transactions on Mathematics, 7, 30-38.

Lee K (2017). Estimation of entropy of the inverse Weibull distribution under generalized progressive hybrid censored data, Journal of the Korean Data \& Information Science Society, 28, 659-668.

Nassar MM and Eissa FH (2004). Bayesian estimation for the exponentiated Weibull model, Сотmunications in Statistics - Theory and Methods, 33, 2343-2362.

Norstrom J (1996). The use of precautionary loss functions in risk analysis, IEEE Transactions on Reliability, 45, 400-403.

Shannon CE (1948). A mathematical theory of communication, The Bell System Technical Journal, 27, 379-423.

Varian HR (1975). A Bayesian Approach to Real Estate Assessment, North-Holland Publishing Company, Amsterdam. 